\title{
Viscosity Measurements of Compressed Liquid Refrigerant Blend R-507A, Using a Vibrating-Wire Technique
}

\author{
Helena M. N. T. Avelino, ${ }^{\dagger}$ João M. N. A. Fareleira,* and Carla M. B. P. Oliveira \\ Centro de Química Estrutural, Instituto Superior Técnico, Universidade Técnica de Lisboa, Av. Rovisco Pais, \\ 1, 1049-001 Lisboa, Portugal
}

\begin{abstract}
The refrigerant blend R-507A (50 wt \% HFC-143a, 50 wt \% HFC-125) is an azeotropic mixture of hydrofluorocarbon refrigerants, 1,1,1-trifluoroethane (HFC-143a) and pentafluoroethane (HFC-125). The paper reports viscosity measurements, performed with a vibrating-wire viscometer, of the refrigerant blend R-507A, at five temperatures in the range (253 to 293$) \mathrm{K}$. The measurements were carried out at pressures from slightly above saturation up to $10 \mathrm{MPa}$, except for the isotherms at $253.26 \mathrm{~K}$ where the maximum pressure was $7.52 \mathrm{MPa}$ and at $263.23 \mathrm{~K}$ where the maximum pressure was $7.09 \mathrm{MPa}$. The overall uncertainty of these measurements is estimated to be $\pm 1.0 \%$. The data obtained were correlated by means of a modified hard-sphere based correlation technique. The root-mean-square deviation, rmsd, of the experimental results from the correlation equations is $0.23 \%$, and their bias is not significant. This correlation method has also been used to interpolate and extrapolate the present results to enable comparisons with measurements performed by other authors of the viscosity of liquid R-507A at different temperatures and pressures.
\end{abstract}

\section{Introduction}

The refrigerant blend R-507A is a mixture of hydrofluorocarbon refrigerants, 1,1,1-trifluoroethane (HFC-143a) and pentafluoroethane (HFC-125) (mass fraction $50 \%$ HFC-143a and $50 \%$ HFC-125). This azeotropic mixture is reported to be nonflammable and to have no ozone depletion potential ${ }^{1}$ and seems to be a candidate fluid for several applications, namely as a replacement for R-502. ${ }^{1}$

Although some of the new refrigerants in practical use are mixtures, experimental viscosity results on compressed liquid refrigerant mixtures are scarce in the literature. ${ }^{2}$ Naturally, this lack of data is itself hindering the assessment of estimation methods. Furthermore, the existence of experimental viscosity data on the pure components is certainly an important piece of information regarding the development of estimation techniques for the viscosity of mixtures. Concerning the blend R-507A, it is therefore noteworthy that, to the knowledge of the authors, only recently has viscosity data on compressed liquid HFC143 a been made available in the literature. ${ }^{3}$

The present measurements of viscosity of the compressed liquid blend R-507A were performed with a vibrating-wire viscometer, at five temperatures in the range (253 to 293) K and pressures up to $10 \mathrm{MPa}$. The results were correlated with the molar volume, as given by the equation of state proposed by Lemmon. ${ }^{4}$ A previously described correlation scheme based on a hard-sphere theory ${ }^{5,6}$ was used for that purpose. The present results were compared with data obtained at thermodynamic saturation conditions by other authors, using the above correlation to perform the small extrapolations of the present data to the saturation pressure. It should be pointed out that viscosity

\footnotetext{
* To whom correspondence should be addressed. E-mail: j.fareleira@ ist.utl.pt.

And Departamento de Engenharia Química, Instituto Superior de Engenharia de Lisboa, R. Conselheiro Emídio Navarro, 1-1950-062 Lisboa, Portugal.

And Universidade Aberta, R. da Escola Politécnica, 147, 1269-001 Lisboa, Portugal.
}

measurements on compressed liquid R-507A have been reported by another author. ${ }^{7}$ These results were obtained for five isotherms, over a temperature range of (300 to 421) K, and at pressures up to about $68 \mathrm{MPa}^{7}$ As no overlapping exists of the temperatures at which those data were obtained with the present measurements, a comparison could only be made by resorting to an extrapolation by means of the mentioned correlation scheme.

An oral description of part of the present work has been presented at the 16th Symposium on Thermophysical Properties. $^{8}$

\section{Experimental Section}

Measurement Method and Equipment. The viscosity measurements were performed with a vibrating-wire viscometer, which has been described previously. ${ }^{3,9,10}$ The measurement method and the ancillary equipment were identical to those used for measuring the viscosity of compressed liquid 1,1,1-trifluoroethane (HFC-143a) and pentafluoroethane (HFC-125). ${ }^{3}$ Temperature stability during a measurement run was typically within $\pm 5 \mathrm{mK}$, and the pressure was measured with an estimated uncertainty of $\pm 0.05 \mathrm{MPa}$. The overall uncertainty of the present viscosity measurements is estimated to be $\pm 1.0 \%$.

The calculation of viscosity requires, in addition to the density of the fluid, knowledge of the density of the wire material $\rho_{\mathrm{s}}$, the wire radius $R$, and the internal damping, $\Delta_{0}$, of the wire. The latter parameters were the same as indicated in our previous publication. $^{3}$

Fluid Samples. The blend R-507A was supplied by Solvay Fluor und Derivate $\mathrm{GmbH}$, under the trade name Solkane. According to the supplier, the mixture, with a fixed composition, has a stated mass purity of $99.5 \%$. The fluid sample was introduced, from the liquid phase, in the measuring cell, without any further treatment, through a $65 \mu \mathrm{m}$ filter (High Pressure Equipment Company, USA). 
Table 1. Experimental Measurements of Viscosity, $\eta$, of R-507A along Five Isotherms at Nominal Temperatures, $T_{n}$, as a Function of Pressure, $p$, Together with Values of Density, $\rho$, Determined from the Equation of State Reported by Lemmon ${ }^{4}$

\begin{tabular}{|c|c|c|c|c|c|c|}
\hline$T_{\mathrm{n}}$ & $p$ & $\rho$ & $\eta$ & $p$ & $\rho$ & $\eta$ \\
\hline $\mathrm{K}$ & $\overline{\mathrm{MPa}}$ & $\mathrm{kg} \cdot \mathrm{m}^{-3}$ & $\overline{\mathrm{mPa} \cdot \mathrm{s}}$ & $\overline{\mathrm{MPa}}$ & $\mathrm{kg} \cdot \mathrm{m}^{-3}$ & $\mathrm{mPa} \cdot \mathrm{s}$ \\
\hline \multirow[t]{11}{*}{253.26} & 2.00 & 1236.3 & 0.2370 & 4.93 & 1248.2 & 0.2479 \\
\hline & 2.01 & 1236.4 & 0.2374 & 4.96 & 1248.3 & 0.2479 \\
\hline & 2.09 & 1236.7 & 0.2377 & 4.97 & 1248.3 & 0.2480 \\
\hline & 2.17 & 1237.0 & 0.2378 & 4.99 & 1248.4 & 0.2482 \\
\hline & 2.18 & 1237.1 & 0.2371 & 5.02 & 1248.5 & 0.2483 \\
\hline & 2.22 & 1237.2 & 0.2379 & 5.07 & 1248.7 & 0.2487 \\
\hline & 3.37 & 1242.0 & 0.2426 & 7.44 & 1257.5 & 0.2574 \\
\hline & 3.38 & 1242.0 & 0.2427 & 7.50 & 1257.7 & 0.2576 \\
\hline & 3.39 & 1242.1 & 0.2427 & 7.62 & 1258.1 & 0.2580 \\
\hline & 3.41 & 1242.2 & 0.2425 & 7.72 & 1258.5 & 0.2587 \\
\hline & 4.90 & 1248.0 & 0.2482 & & & \\
\hline \multirow[t]{9}{*}{263.23} & 1.99 & 1201.6 & 0.2076 & 3.72 & 1210.0 & 0.2140 \\
\hline & 2.02 & 1201.7 & 0.2074 & 3.78 & 1210.3 & 0.2140 \\
\hline & 2.07 & 1202.0 & 0.2081 & 4.69 & 1214.4 & 0.2177 \\
\hline & 2.12 & 1202.2 & 0.2077 & 4.99 & 1215.8 & 0.2192 \\
\hline & 3.66 & 1209.7 & 0.2139 & 5.01 & 1215.9 & 0.2195 \\
\hline & 3.67 & 1209.7 & 0.2137 & 7.00 & 1224.5 & 0.2258 \\
\hline & 3.67 & 1209.7 & 0.2139 & 7.05 & 1224.7 & 0.2257 \\
\hline & 3.68 & 1209.8 & 0.2138 & 7.19 & 1225.3 & 0.2262 \\
\hline & 3.68 & 1209.8 & 0.2137 & 7.22 & 1225.4 & 0.2270 \\
\hline \multirow[t]{11}{*}{273.24} & 2.15 & 1165.5 & 0.1827 & 3.50 & 1173.4 & 0.1878 \\
\hline & 2.16 & 1165.6 & 0.1825 & 5.03 & 1181.7 & 0.1923 \\
\hline & 2.19 & 1165.7 & 0.1826 & 5.04 & 1181.7 & 0.1925 \\
\hline & 2.19 & 1165.7 & 0.1823 & 5.06 & 1181.9 & 0.1925 \\
\hline & 2.23 & 1166.0 & 0.1829 & 7.36 & 1193.4 & 0.2011 \\
\hline & 2.26 & 1166.2 & 0.1829 & 7.45 & 1193.8 & 0.2008 \\
\hline & 2.27 & 1166.2 & 0.1829 & 7.45 & 1193.8 & 0.2008 \\
\hline & 3.45 & 1173.1 & 0.1878 & 7.52 & 1194.1 & 0.2015 \\
\hline & 3.45 & 1173.1 & 0.1876 & 9.71 & 1204.1 & 0.2103 \\
\hline & 3.47 & 1173.2 & 0.1877 & 9.88 & 1204.9 & 0.2106 \\
\hline & 3.48 & 1173.3 & 0.1873 & 9.88 & 1204.9 & 0.2104 \\
\hline \multirow[t]{14}{*}{283.34} & 2.00 & 1124.1 & 0.1592 & 3.86 & 1137.4 & 0.1655 \\
\hline & 2.06 & 1124.6 & 0.1592 & 3.88 & 1137.5 & 0.1657 \\
\hline & 2.12 & 1125.0 & 0.1591 & 4.97 & 1144.6 & 0.1693 \\
\hline & 2.16 & 1125.3 & 0.1591 & 5.00 & 1144.8 & 0.1693 \\
\hline & 2.22 & 1125.8 & 0.1593 & 5.03 & 1145.0 & 0.1696 \\
\hline & 2.23 & 1125.9 & 0.1592 & 5.04 & 1145.1 & 0.1695 \\
\hline & 2.23 & 1125.9 & 0.1596 & 7.60 & 1160.2 & 0.1780 \\
\hline & 2.25 & 1126.0 & 0.1596 & 7.64 & 1160.4 & 0.1783 \\
\hline & 2.26 & 1126.1 & 0.1595 & 7.68 & 1160.6 & 0.1784 \\
\hline & 2.27 & 1126.2 & 0.1597 & 9.75 & 1171.6 & 0.1851 \\
\hline & 3.72 & 1136.5 & 0.1650 & 9.86 & 1172.2 & 0.1854 \\
\hline & 3.77 & 1136.8 & 0.1651 & 9.90 & 1172.4 & 0.1857 \\
\hline & 3.77 & 1136.8 & 0.1654 & 9.92 & 1172.5 & 0.1857 \\
\hline & 3.82 & 1137.1 & 0.1654 & 9.93 & 1172.5 & 0.1856 \\
\hline \multirow{10}{*}{293.33} & 1.92 & 1079.1 & 0.1394 & 5.00 & 1105.9 & 0.1506 \\
\hline & 1.94 & 1079.3 & 0.1392 & 5.03 & 1106.1 & 0.1506 \\
\hline & 1.97 & 1079.6 & 0.1396 & 7.51 & 1123.8 & 0.1585 \\
\hline & 2.02 & 1080.1 & 0.1394 & 7.56 & 1124.2 & 0.1584 \\
\hline & 3.57 & 1094.3 & 0.1450 & 7.54 & 1124.0 & 0.1584 \\
\hline & 3.69 & 1095.3 & 0.1454 & 9.77 & 1137.9 & 0.1667 \\
\hline & 3.72 & 1095.3 & 0.1455 & 9.81 & 1138.2 & 0.1669 \\
\hline & 3.75 & 1095.8 & 0.1457 & 9.85 & 1138.4 & 0.1666 \\
\hline & 4.91 & 1105.2 & 0.1500 & 9.88 & 1138.6 & 0.1669 \\
\hline & 4.91 & 1105.2 & 0.1503 & 9.93 & 1138.9 & 0.1672 \\
\hline
\end{tabular}

\section{Results}

The viscosity of compressed liquid R-507A was measured along five isotherms at temperatures between (253 and 293) K. The measurements were performed at pressures from slightly above saturation up to $10 \mathrm{MPa}$, except for the isotherms at $253.26 \mathrm{~K}$ where the maximum pressure was $7.52 \mathrm{MPa}$ and at 263.23 K where the maximum pressure was 7.09 MPa. The viscosity measurements were performed at temperatures close to the nominal temperatures, $T_{\mathrm{n}}$, shown in Table 1 . The raw viscosity data were subsequently corrected to the corresponding value of $T_{\mathrm{n}}$ through linear interpolation, which did not exceed $0.02 \mathrm{~K}$. The density values, required to obtain the viscosity from the working equations, were calculated using the equation of state published by Lemmon. ${ }^{4}$ According to the author, the deviations of the pseudopure fluid equation of state from the full mixture model ${ }^{11}$ do not exceed $\pm 0.1 \%$, except in the critical region. ${ }^{4}$

\section{Discussion}

Data Correlation. All the measurements of the viscosity of R-507A were correlated with the fluid density using the same method that has been applied to its pure components, ${ }^{3} \mathrm{HFC}$ 143a and HFC-125. The correlation technique is a heuristic development of the application of the kinetic theory of a dense hard-sphere fluid to the van der Waals model of a liquid ${ }^{6}$ and has been proposed by Li et al. ${ }^{5}$ This correlation uses a reduced dimensionless viscosity, $\eta^{*}$, which, using base SI units, is defined by

$$
\eta^{*}=6.035 \cdot 10^{8} \eta V^{2 / 3} /(M R T)^{1 / 2}
$$

where $\eta$ is the viscosity; $M$ is the molar mass; $T$ is the temperature; $V$ is the molar volume; and $R$ is the gas constant. Using the procedure described elsewhere, ${ }^{3}$ the data are correlated by a single equation of the form

$$
\ln \eta^{*}=\sum_{j=0}^{n} a_{j}\left(\frac{V_{0}}{V-V_{0}}\right)^{-j}
$$

where $V_{0}$ is a slightly temperature-dependent characteristic volume and $n=4$ for the present results. The choice of the value for $n$ has been made from the observation that the utilization of a higher number of parameters in eq 2 did not produce significantly smaller values for the statistical parameters AAD, rmsd, or bias. This method enables the optimization of an expression of the type of eq 2, dedicated specifically to one fluid or to one set of measurements, and it has been applied before to several liquids (2,2,4-trimethylpentane $,{ }^{12} \mathrm{HFC}-134 \mathrm{a},{ }^{13}$ and toluene ${ }^{14,15}$ ).

In the present work, the blend R-507A was treated as a pseudopure fluid. Hence a reference value for $V_{0}$, at an arbitrary reference temperature, $T_{\text {ref }}$, was calculated, following a procedure previously applied to refrigerants by Teja et al..$^{16}$ Assuming that $V_{0}$ is, at this temperature, equivalent to the volume of closepacking of hard spheres, ${ }^{6}$ it was calculated from the value of the hard-sphere diameter, $\sigma_{\mathrm{HS}}$

$$
V_{0}=\left(\sigma_{\mathrm{HS}}^{3} / 2^{0.5}\right) N_{\mathrm{A}}
$$

In eq 3, $\sigma_{\mathrm{HS}}$ is the hard-sphere diameter and $N_{\mathrm{A}}$ is Avogadro's constant. The diameter, $\sigma_{\mathrm{HS}}$, for R-507A was obtained from the Lennard-Jones parameters, $\sigma_{\mathrm{LJ}}$ and $\epsilon_{\mathrm{LJ}}$, reported by Nabizadeh and Mayinger, ${ }^{17}$ making use of a correlation proposed by Hammonds and Heyes. ${ }^{18}$

The reference temperature for R-507A was chosen to be $273.24 \mathrm{~K}$. The values of $V_{0}$ at each of the other nominal temperatures, $T_{\mathrm{n}}$, at which the present measurements were performed have been obtained as described before. ${ }^{3}$ The molar volumes of R-507A were calculated from the equation of state developed by Lemmon. ${ }^{4}$ In Table 2, the values of $V_{0}$ at all the nominal temperatures of the present measurements are shown, including the reference temperature.

The values of $V_{0}$ in Table 2 were calculated as described before $^{3}$ and are represented by the empirical relation

$$
V_{0} \cdot 10^{6} /\left(\mathrm{m}^{3} \cdot \mathrm{mol}^{-1}\right)=a+b(T / \mathrm{K})+c(T / \mathrm{K})^{2}
$$

The fitting parameters $a, b$, and $c$ are given in Table 3 . 
Table 2. Characteristic Volumes, $V_{0}$, for R-507A at the Nominal Temperatures of the Present Viscosity Measurements

\begin{tabular}{cc}
\multicolumn{2}{c}{$\mathrm{R}-507 \mathrm{~A}$} \\
\cline { 2 - 3 }$T_{\mathrm{n}} / \mathrm{K}$ & $10^{6} V_{0} \mathrm{~m}^{3} \cdot \mathrm{mol}^{-1}$ \\
254.26 & 51.80 \\
263.23 & 51.58 \\
273.24 & 51.35 \\
283.34 & 51.07 \\
293.33 & 50.77
\end{tabular}

Table 3. Fitting Parameters of Equation 4

\begin{tabular}{cc}
\hline & $\mathrm{R}-507 \mathrm{~A}$ \\
\hline$a$ & 47.02 \\
$b$ & $5.747 \cdot 10^{-2}$ \\
$c$ & $-1.524 \cdot 10^{-4}$
\end{tabular}

Table 4. Fitting Parameters of Equation 2 and the Corresponding Average Absolute Deviation, AAD, Bias, and Root-Mean-Square Deviation, rmsd, of the Present Data from Equation 2, Calculated According to Equations 5 to 7

\begin{tabular}{lc}
\hline & $\mathrm{R}-507 \mathrm{~A}$ \\
\hline$a_{0}$ & 1.73484 \\
$a_{1}$ & 0.20853 \\
$a_{2}$ & 0.19403 \\
$a_{3}$ & 0.12037 \\
$a_{4}$ & -0.05353 \\
$\mathrm{AAD} / \%$ & 0.19 \\
$\mathrm{bias} / \%$ & 0.00 \\
$\mathrm{rmsd} / \%$ & 0.23
\end{tabular}

The coefficients, $a_{i}$, of eq 2 that best represent the present results of the viscosity measurements of R-507A, using the values of $V_{0}$ shown in Table 2, are listed in Table 4.

The relative deviations of our measurements from eq 2 are shown in Figure 1. The maximum deviation does not exceed \pm $0.56 \%$; the corresponding average absolute deviation, AAD, of the experimental points from the fitting is $0.19 \%$; and their bias is not significant (being several orders of magnitude smaller than the AAD). The root-mean-square deviation, rmsd, of the experimental results from the correlations is $0.23 \%$. The quantities $\mathrm{AAD}$, bias, and rmsd used were defined as follows:

$$
\begin{gathered}
\mathrm{AAD} / \%=\frac{100}{N} \sum_{i}^{N}\left|\frac{\eta_{\text {exptl }, i}}{\eta_{\text {calcd }, i}}-1\right| \\
\mathrm{bias} / \%=\frac{100}{N} \sum_{i}^{N}\left(\frac{\eta_{\text {expt }, i}}{\eta_{\text {calcd }, i}}-1\right) \\
\mathrm{rmsd} / \%=100\left[\frac{1}{N} \sum_{i}^{N}\left(\frac{\eta_{\text {exptl }, i}}{\eta_{\text {corr }, i}}-1\right)^{2}\right]^{1 / 2}
\end{gathered}
$$

where $\eta_{\text {exptl, } i}$ is the $i$ th viscosity experimental datum; $\eta_{\text {corr }, i}$ is the corresponding value of the viscosity calculated by means of the correlation eq 2 ; and $N$ is the number of experimental data in the set of measurements used to define the correlation equation.

Comparison with Literature Data. For comparison purposes, the only viscosity measurements on compressed liquid R-507A that could be found in the literature have been reported by Laesecke. ${ }^{7}$ Those data were obtained using a torsional crystal viscometer, along five isotherms, over a temperature range of approximately (300 to 421$) \mathrm{K}$ and at pressures up to about 68 $\mathrm{MPa}$. The uncertainty of such measurements is, in general, estimated $^{7}$ not to exceed $\pm 2 \%$. As the temperature ranges of those measurements and of the present results do not overlap, a comparison can only be made with resort to data extrapolation. Moreover, most of the data published by Laesecke ${ }^{7}$ were

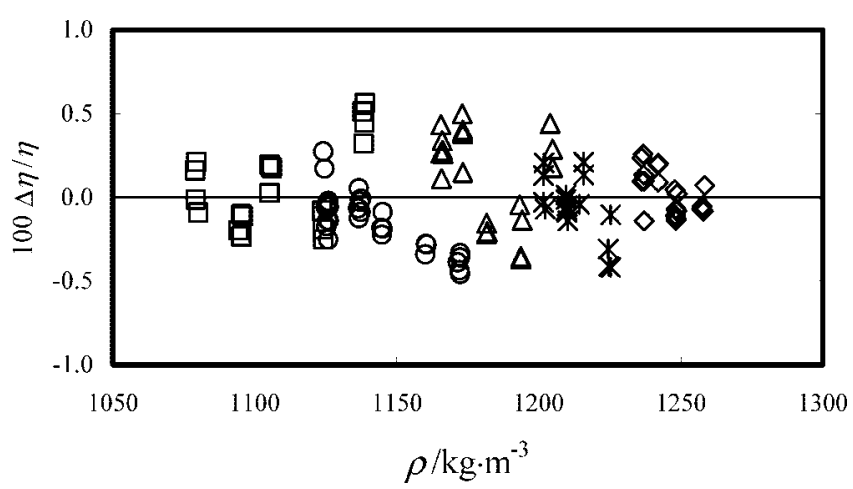

Figure 1. Relative deviation, $\Delta \eta / \eta=\eta_{\text {exptl, } i} / \eta_{\text {corr }, i}-1$, of the present viscosity measurements of R-507A (Table 1), $\eta_{\text {exptl }, i}$, from the correlation of the present experimental results given by equation $2, \eta_{\mathrm{corr}, i}$, as a function of density along the isotherms: $\diamond, 253.26 \mathrm{~K} ; *, 263.23 \mathrm{~K} ; \Delta, 273.24 \mathrm{~K} ; \bigcirc$, 283.22 K; $\square, 293.33 \mathrm{~K}$.

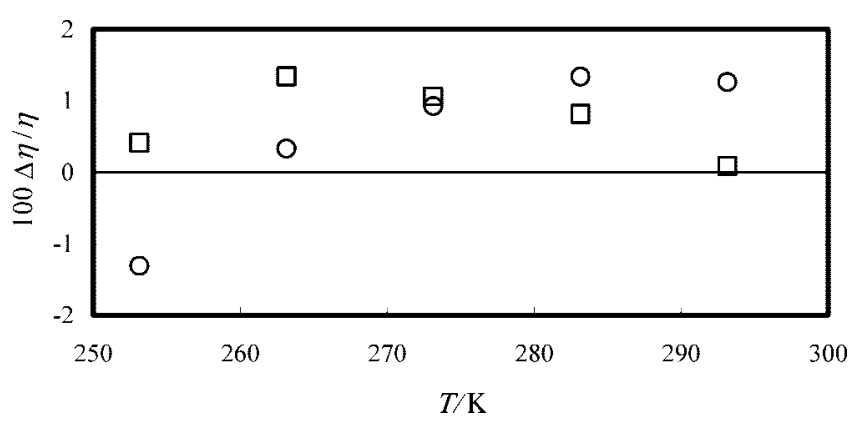

Figure 2. Deviation, $\Delta \eta / \eta=\eta_{\mathrm{lit}} / \eta_{\mathrm{corr}}-1$, of literature viscosity data on $\mathrm{R}-507 \mathrm{~A}, \eta_{\text {lit }}$, from the correlation of the present experimental results given by eq $2, \eta_{\text {corr }}$, as a function of temperature: $\bigcirc$, ref $19 ; \square$, refs 20 and 21 .

obtained at supercritical or near critical temperatures. As a consequence, only the compressed liquid R-507A viscosity data published by that author at approximately $300 \mathrm{~K}$ were compared with the present results. For this purpose, the latter were extrapolated by means of the correlation eqs 2 and 4, with the parameters given in Tables 3 and 4. Only two data points of the isotherm obtained by Laesecke ${ }^{7}$ are within the pressure range of the present work. Those points deviate from the extrapolation of the present data by $-0.22 \%$ at $3.6 \mathrm{MPa}$ and $+0.63 \%$ at 6.6 $\mathrm{MPa}$, which is an excellent agreement. Moreover, an extrapolation of eq 2 along the isotherm at about $300 \mathrm{~K}$ up to a pressure of approximately $67 \mathrm{MPa}$ shows that the absolute deviations of the data published by Laesecke ${ }^{7}$ do not exceed $1.9 \%$, which is still within the nominal uncertainty of the experimental method.

Measurements of the kinematic viscosity of liquid R-507A, at temperatures overlapping those of the present work, were performed along the saturation line, by Fröba et al. ${ }^{19}$ Those results, obtained with a surface light scattering technique, have an estimated uncertainty of $\pm 2 \%$ and, for comparison with the present data, have been converted to dynamic viscosities, using the density values reported by the same authors. ${ }^{19}$ Recently, these results have been recalculated by Fröba using a more rigorous description of the liquid-vapor interface. ${ }^{20,21}$ The deviations of both sets of data from the correlation represented by eq 2 , with the parameters given in Table 4, along the temperature range of the present results are shown in Figure 2. This comparison required the extrapolation of the present results to the saturation pressure by means of eq 2 . The comparison shows that the results first reported by Fröba et al. ${ }^{19}$ have deviations within $\pm 1.3 \%$ from the present values, while the corrected data ${ }^{20,21}$ deviate from (0.01 to 1.32$) \%$. Therefore, 
it may be concluded that, over the temperature range of our measurements, the deviations are well within the combined uncertainty of the experimental methods.

It is interesting to note that the viscosity of R-507A is predicted in excellent agreement with the present results, as a mole fraction average of the viscosity of its pure components at the same temperature and pressure, as calculated by the corresponding correlation equations of the experimental data published previously ${ }^{3}$

$$
\eta_{\text {mixt }}(T, p)=x_{i} \eta_{i}(T, p)+x_{j} \eta_{j}(T, p)
$$

The deviations of the values calculated by eq 8 from the experimental data shown in Table 1 are within $(-0.56$ to +0.84$)$ $\%$ and are characterized by an AAD of $0.33 \%$ and a bias of $+0.19 \%$, where the following definitions have been used:

$$
\begin{aligned}
& \mathrm{AAD} / \%=\frac{100}{N} \sum_{i}^{N}\left|\frac{\eta_{\text {calcd }, i}}{\eta_{\text {expt }, i}}-1\right| \\
& \text { bias } / \%=\frac{100}{N} \sum_{i}^{N}\left(\frac{\eta_{\text {calcd }, i}}{\eta_{\text {expt }, i}}-1\right)
\end{aligned}
$$

where $\eta_{\text {exptl, } i}$ is the $i$ th viscosity experimental datum of the present work (see Table 1); $\eta_{\text {calcd }, i}$ is the corresponding value of the viscosity calculated by means of eq 8 ; and $N$ is the number of experimental points in Table 1. It is noteworthy that the correlation eq 8 has been $u_{\text {sed }}{ }^{22}$ as a reference for calculating the viscosity of mixing.

\section{Conclusions}

New viscosity measurements of compressed liquid R-507A in the temperature range ( 253 to 293 ) $\mathrm{K}$ and pressures up to 10 MPa have been presented. To the knowledge of the authors, the present results are the first set of viscosity data of compressed liquid $\mathrm{R}-507 \mathrm{~A}$, over that temperature range. The overall uncertainty of the results, obtained using a vibrating-wire instrument, is estimated not to exceed $\pm 1.0 \%$. The experimental results were fitted successfully with a correlation scheme based on a hard-sphere model. This was used for extrapolating the present data to the saturation line and to other conditions of temperature and pressure, to enable comparisons with literature data. Good agreement has been found with two sets of recent viscosity measurements of liquid R-507A.

\section{Acknowledgment}

The authors are grateful to Prof. U.V. Mardolcar (Department of Physics, Instituto Superior Técnico, Lisbon, Portugal) and SolvayFluor und Derivate GmbH, Germany, for having kindly supplied the sample of R-507A. The authors also thank Dr.-Ing. A. P. Fröba (Lehrstuhl für Technische Thermodynamik, Friedrich-AlexanderUniversität, Erlangen-Nürnberg, Germany) for calling their attention to the existence of corrected data sets on the viscosity of R-507A, obtained by the surface light scattering method.

\section{Literature Cited}

(1) Solvay Fluor und Derivate GmBh, Technical information,http://www. solvay-fluor.com/chemicals/solkane_507, accessed May 2006.
(2) Klein, S. A.; McLinden, M. O.; Laesecke, A. An improved extended corresponding states method for estimation of viscosity of pure refrigerants and mixtures. Int. J. Refrig. 1997, 20, 208-217.

(3) Avelino, H. M. N. T.; Fareleira, J. M. N. A.; Oliveira, C. M. B. P. Viscosity of Compressed Liquid 1,1,1-Trifluoroethane (HFC-143a) and Pentafluoroethane (HFC-125). J. Chem. Eng. Data 2006, 51, 16721677.

(4) Lemmon, E. W. Pseudo-Pure Fluid Equations of State for the Refrigerant Blends R410A, R-404A, R507A, and R-407C. Int. J. Thermophys. 2003, 24, 991-1006.

(5) Li, S. F. Y.; Trengove, R. D.; Wakeham, W. A.; Zalaf, M. The Transport Coefficients of Polyatomic Liquids. Int. J. Thermophys. 1986, 7, 273-284.

(6) Dymond, J. H. Hard-Sphere Theories of Transport Properties. Chem. Soc. Rev. 1985, 14, 317-356.

(7) Laesecke, A. Viscosity measurements and model comparisons for the refrigerant blends R-410A and R-507A. ASHRAE Trans: Symposia 2004, 503-521.

(8) Avelino, H. M. N. T.; Fareleira, J. M. N. A.; Oliveira, C. M. B. P. Viscosity measurements of compressed liquid refrigerant blend R-507A, 16th Symposium on Thermophysical Properties, Thermo International, Boulder, Co, USA, 30 July-4 August, 2006.

(9) Correia da Mata, J. L. G.; Fareleira, J. M. N. A.; Oliveira, C. M. B. P.; Wakeham, W. A.; Caetano, F. J. P. A New Instrument to Perform Simultaneous Measurements of Density and Viscosity of Fluids by a Dual Vibrating-Wire Technique. High Temp.- High Pressures 2001, 33, 669-676.

(10) Caetano, F. J. P.; Correia da Mata, J. L. G.; Fareleira, J. M. N. A.; Oliveira, C. M. B. P.; Wakeham, W. A. Viscosity Measurements of Liquid Toluene at Low Temperatures Using a Dual Vibrating-Wire Technique. Int. J. Thermophys. 2004, 25, 1-11.

(11) Lemmon, E. W.; Jacobsen, R. T. Equations of State for Mixtures of R-32, R-125, R-132, R-143a and R-152a. J. Phys. Chem. Ref. Data 2004, 33, 593-620.

(12) Pádua, A. A. H.; Fareleira, J. M. N. A.; Calado, J. C. G.; Wakeham, W. A. Density and Viscosity Measurements of 2,2,4-Trimethylpentane (Isooctane) from 198 to $348 \mathrm{~K}$ and up to $100 \mathrm{MPa}$. J. Chem. Eng. Data 1996, 41, 1488-1494.

(13) Pádua, A. A. H.; Fareleira, J. M. N. A.; Calado, J. C. G.; Wakeham, W. A. Density and Viscosity Measurements of 1,1,1,2-Tetrafluoroethane (HFC-134a) from 199 to $298 \mathrm{~K}$ and up to $100 \mathrm{MPa}$. J. Chem. Eng. Data 1996, 41, 731-735.

(14) Avelino, H. M. N. T.; Fareleira, J. M. N. A.; Wakeham, W. A. Simultaneous Measurement of the Density and Viscosity of Compressed Liquid Toluene. Int. J. Thermophys. 2003, 24, 323-336.

(15) Assael, M. J.; Avelino, H. M. N. T.; Dalaouti, N. K.; Fareleira, J. M. N. A.; Harris, K. R. Reference Correlation for the Viscosity of Liquid Toluene from 213 to $373 \mathrm{~K}$ at Pressures to $250 \mathrm{MPa}$. Int. J. Thermophys. 2001, 22, 789-799.

(16) Teja, A. S.; Smith, R. L.; King, R. K.; Sun, T. F. Correlation and Prediction of the Transport Properties of Refrigerants Using Two Modified Rough Hard-Sphere Models. Int. J. Thermophys. 1999, 20, 149-161.

(17) Nabizadeh, H.; Mayinger, F. Viscosity of Gaseous R404A, R407C, R410A, and R507. Int. J. Thermophys. 1999, 20, 777-790.

(18) Hammonds, K. D.; Heyes, D. M. Transport-Coeficients of Model Simple Liquids. A Molecular-Dynamics Study and Effective HardSphere. J. Chem. Soc. Faraday Trans. 1988, 84, 705-725.

(19) Fröba, A. P.; Will, S.; Leipertz, A. Thermophysical Properties of Binary and Ternary Fluid Mixtures from Dynamic Light Scattering. Int. J. Thermophys. 2001, 22, 1349-1368.

(20) Fröba, A. P. Ph.D. Thesis, Friedrich-Alexander-Universität, ErlangenNürnberg, 2002.

(21) Fröba, A. P.,personal communication, 2006.

(22) Aminabhavi, T. M.; Gopalkrishna, B. Densities, Viscosities and Refractive Indices of the Binary Mixtures of Bis(2-methoxyethyl) Ether with 1-propanol, 1-butanol, 2-methyl-1-propanol, and 2-methyl-2propanol. J. Chem. Eng. Data 1994, 39, 865-867.

Received for review June 15, 2007. Accepted October 02, 2007. The present work has been supported by Fundação para a Ciência e a Tecnologia (Portugal) under the PRAXIS and POCTI programs, with European Union funds (FEDER) and National funds.

JE700345B 